Article

\title{
Determining of the Joint Roughness Coefficient (JRC) of Rock Discontinuities Based on the Theory of Random Fields
}

\author{
Elias Gravanis and Lysandros Pantelidis * \\ Department of Civil Engineering and Geomatics, Cyprus University of Technology, 2-8 Saripolou str, \\ 3603 Limassol, Cyprus \\ * Correspondence: lysandros.pantelidis@cut.ac.cy
}

Received: 29 May 2019; Accepted: 2 July 2019; Published: 4 July 2019

\begin{abstract}
This work intends to embed the estimation of the joint roughness coefficient (JRC) in the framework of random fields. The random field method is a probabilistic approach which involves modeling of the spatial variability of the pertinent physical quantities as a fundamental part of the (assumed) underlying probabilistic structure. Although this method is one of higher complexity in regard of the presumed background knowledge, it encodes naturally subtler information about the rock surface roughness. It is noted that, the proposed random field approach considers automatically the scale of the problem (no correction factor is needed), whilst the JRC estimates appear to be more stable (compared to those derived from $Z_{2}$ or $\mathrm{SF}$ ) in the sense that images of the same profile but of different quality give similar results for its roughness. The present work could also be useful in advanced probabilistic rock slope stability analysis based on random fields. In such a case, the required spatial correlation length $\theta$ can be obtained by the proposed $\theta=145.5 \mathrm{\sigma} / \mathrm{JRC}$ relationship ( $\sigma=$ variance of the profile). The JRC can be obtained through tilt tests, push or pull tests, or matching roughness profiles, whilst $\sigma$ can be obtained from inspection of the digitized profile.
\end{abstract}

Keywords: joint roughness coefficient; Barton-Bandis criterion; random fields; spatial correlation length; Fourier analysis; probabilistic analysis

\section{Introduction}

In 1955, the American Standards Association [1] suggested the following indices for giving numerical values to certain components of surface roughness:

$$
\begin{gathered}
C L A=\frac{1}{L} \int_{x=0}^{x=L}|y| d x, \\
Z_{1}=R M S=\frac{1}{L} \sqrt{\int_{x=0}^{x=L} y^{2} d x,}
\end{gathered}
$$

where, $C L A$ and $Z_{1}$ stands for center line average and root mean square respectively, $L$ is the distance over which the average is taken, is the amplitude of the roughness about the center line and $d x$ is the small constant distance between two adjacent amplitude readings.

A few years later, Myers [2] reported that although many different surfaces have the same RMS (or $C L A$ ) value, their geometrical properties differ greatly, suggesting the following three new characteristics:

$$
Z_{2}=\frac{1}{L} \sqrt{\int_{x=0}^{x=L}\left(\frac{d y}{d x}\right)^{2} d x}
$$




$$
Z_{3}=\frac{1}{L} \sqrt{\int_{x=0}^{x=L}\left(\frac{d^{2} y}{d x^{2}}\right)^{2} d x}
$$

and

$$
\mathrm{Z}_{4}=\frac{\sum\left(\Delta x_{i}\right)_{p}-\sum\left(\Delta x_{i}\right)_{n}}{L}
$$

where, $L=\Sigma\left(\Delta x_{i}\right)_{p}+\Sigma\left(\Delta x_{i}\right)_{n}$ is the total profile distance, $x_{i}$ is segment of $L$ and the subscripts $p$ and $n$ mean positive and negative slope respectively. In this respect, the experimental work carried out by Myers [2] showed that the most useful characteristic in predicting friction was the $Z_{2}$ one.

In 1979, Tse and Cruden [3] put again the above Z-characteristics into question. The objective was a convincing correlation with the joint roughness coefficient (JRC). In this respect, the ten standards profiles of Barton and Choubey [4] were used (these profiles are shown in the Appendix A). The JRC coefficient is of particular importance for calculating the peak shear strength of rock surfaces through Barton and Choubey's empirical equation:

$$
\tau_{\text {peak }}=\sigma_{n} \tan \left(\phi_{r}+J R C \log \frac{J C S}{\sigma_{n}}\right),
$$

where, $\tau_{\text {peak }}$ is the maximum shear strength, $\sigma_{n}$ is normal stress, $\varphi_{r}$ is the residual friction angle of discontinuity and JCS is the joint compressive strength. JCS can be estimated using the Schmidt hammer [5]. In addition to the Z-characteristics, in their comparison, Tse and Cruden [3] used the following statistical indices:

$$
\begin{gathered}
M S V=\frac{1}{L} \int_{x=0}^{x=L} y^{2} d x, \\
A C F=\frac{1}{L} \int_{x=0}^{x=L} y(x) y(x+\Delta x) d x,
\end{gathered}
$$

and

$$
S F=\int_{0}^{L}(y(x)-y(x+\Delta x))^{2} d x,
$$

where, $y(x)$ is the amplitude of asperity height at the distance, $x$, along the length, $L$, and $\Delta x$ is a constant distance lag. MSV, ACF and SF stand for mean square values, autocorrelation function and structure function respectively, the latter has been proposed by Sayles and Thomas [6]. Tse and Cruden [3] confirmed that, among all $Z$-characteristics, the $Z_{2}$ one performed better, whilst for the $S F$ index they found that it was as good as $Z_{2}$. Indeed, they gave JRC- $\log Z_{2}$ and JRC-logSF relationships having R-squared values as high as 0.972 and 0.968 respectively. $\mathrm{Yu}$ and Vayssade [7] reported an infinitesimal improvement based on the JRC $-\sqrt{Z_{2}}$ and JRC $-\sqrt{S F}$ relationships. It is mentioned that, Tse and Cruden [3] used a laborious procedure to eliminate possible errors, something that apparently increased the R-squared value of their correlations. The authors attempted to reproduce the JRC- $\log Z_{2}$ relationship and they found that:

$$
\mathrm{JRC}=48.707 \log Z_{2}+37.912,
$$

with $R^{2}=0.933$. The original Barton and Choubey's [4] image depicting the ten standard JRC profiles was used. The graph of Figure 1 was drawn based on 1024 sampling points for each of these profiles after subtracting the linear trend. The digitalization was done with Wolfram Mathematica. It was only verified that the digitized images coincide visually with Barton and Choubey's profiles having involved no further fine tuning of the digitization. Apparently, the result depends on both sampling and the quality of image. This is in keeping with the very notion of the standard profiles. Besides, the goal of this paper is not a refined JRC- $\log Z_{2}$ (or similar) relationship which will just increase $\mathrm{R}^{2}$ by a few second decimal units. It is noted that, the various sources of error are discussed in Gao and Wong [8]. 


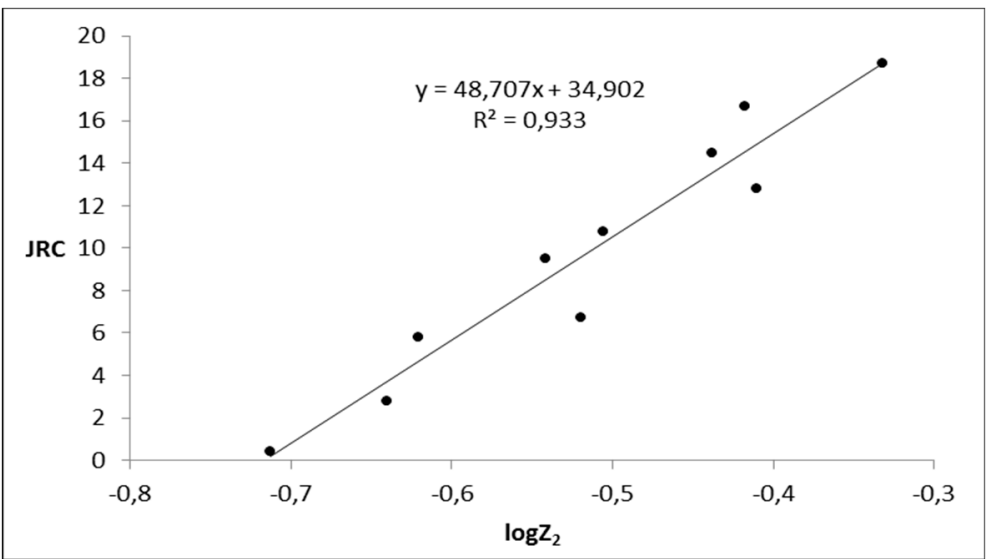

Figure 1. JRC- $\log Z_{2}$ relationship for the ten standard JRC profiles of Barton and Choubey [4].

Since Tse and Cruden [3], numerous other researchers have either evaluated the existing methods for estimating the JRC coefficient or proposed new ones [7,9-22], e.g., Yang et al. [12] and Yu and Vayssade [7] found $R^{2}=0.986$ and 0.910 for the JRC- $\log Z_{2}$ relationship respectively. However, the $Z_{2}$ characteristic remains the most popular one, apparently due to its simplicity and efficiency. A review of these methods is out of scope of the present paper. In this respect, the authors suggest the works done by Li and Zhang [13] and Li and Huang [14]. Instead, the present work intends to embed the estimation of the joint roughness coefficient (JRC) in the framework of the random fields. The random field method is a probabilistic approach to geotechnical problems which involves modeling the spatial variability of the pertinent physical quantities as a fundamental part of the (assumed) underlying probabilistic structure.

\section{Methods}

The geometric description uses the information contained in the details of the shape of the curve to provide a measure of roughness in a deterministic way. There are many levels of complexity in the geometric description of the profile going all the way to fractal geometry. A recent literature review of empirical equations for estimating JRC by using the fractal dimension $D$ has been offered by Li and Huang [14]. As mentioned above, an elementary and attractive choice is to use the quantity $Z_{2}$ defined by Equation (3).

On the other hand, in statistical description, roughness is understood as the estimated value of a certain statistic, treating the profile as a collection of events that obey an underlying probabilistic structure. The autocorrelation function (ACF; Equation (8)) is an example of such a statistic. For example, for zero correlation distance $(\Delta x=0)$, the correlation function computes an estimate of the variance $\sigma^{2}$ of the profile regarding the height $y$ at each point $x$ as a random variable with a fixed variance.

In Tse and Cruden [3], the structure function (SF) defined by Equation (9) has been correlated with the JRC value of the ten standard profiles, which treated $\Delta x$ as fixed and equal to the length of the interval between two successive sampling points. This showed that it is as good an index as $Z_{2}$. This is not surprising because if $\Delta x$ is the sampling interval, the discrete version of Equations (8) and (9) essentially coincide. Still, the statistical information contained in the ACF as a function over its entire natural range $(-L / 2, L / 2)$ has neither been exploited nor been put in a statistical framework. This is done herein with the statistical description of roughness based on random fields. In this respect, a profile as a whole is regarded as a single realization of an underlying probabilistic structure where the function $A C F(\triangle x)$ over the whole of its natural range $(-L / 2, L / 2)$ is assumed to have a fixed and pre-defined form. This form is denoted by $C(\Delta x)$ and called the variance function of the random field [23]. The exact definitions are given below. 
A function y over $-\infty \leq x \leq \infty$ is a (stationary Gaussian) random field if every number $\mathrm{y}(x)$ is a Gaussian random variable with (constant) mean value $\mu$ and point-variance $\sigma^{2}$, i.e.,

$$
\mathrm{E}[\mathrm{y}(\mathrm{x})]=\mu, \mathrm{E}\left[\mathrm{y}(\mathrm{x})^{2}\right]=\mu^{2}+\sigma^{2},
$$

where, $\mathrm{E}[\ldots$... ] denotes the expectation value of the quantity inside the brackets over a large number of realizations of the random field, and every two random variables $\mathrm{y}(\mathrm{x}), \mathrm{y}(\mathrm{x}+\Delta \mathrm{x})$ (associated with two different positions $\mathrm{x}, \mathrm{x}+\Delta \mathrm{x}$ along the interval) are correlated in a given manner, i.e.,

$$
\mathrm{E}[\mathrm{y}(\mathrm{x}) \mathrm{y}(\mathrm{x}+\Delta \mathrm{x})]=\mu^{2}+\mathrm{C}(\Delta \mathrm{x})
$$

These define completely the Gaussian random field. The variance function $C(\Delta x)$ can be chosen in a variety of ways depending on the nature of the quantity being attempted to model. Clearly, $C(0)=\sigma^{2}$ by Equations (11) and (12). Therefore, defining the normalized variance function:

$$
\rho(\Delta \mathrm{x})=\mathrm{C}(\Delta \mathrm{x}) / \mathrm{C}(0),
$$

which satisfies $\rho(0)=1$. The coordinates in the description of the profiles are chosen so that the mean value $\mu$ is zero, so there is no difference between the autocorrelation and variance function of the profile.

A very convenient choice is the Gaussian variance function:

$$
\rho(\Delta \mathbf{x})=\exp \left[-\pi(\Delta \mathbf{x})^{2} / \theta^{2}\right]
$$

The spatial correlation length $\theta$ is defined by

$$
\theta=\int_{-\infty}^{\infty} \rho(\Delta x) d(\Delta x)=2 \int_{0}^{\infty} \rho(\Delta x) d(\Delta x)
$$

(e.g., see $[23,24])$ noting that the variance function is even.

It is also noted, that the correlation length is related to the value of the derivatives of the variance function at zero. In particular the value of the second derivative reads:

$$
-\rho^{\prime \prime}(0)=2 \pi / \theta^{2}
$$

The integral definition above defines, for any variance function, a macro-scale, while the derivative definition defines a micro-scale. These definitions are usual in other areas where correlation functions are used, e.g., in the theory of turbulence [25]. In general, if the micro-scale can be defined at all, they could be entirely different. The fact that they are both related to the single length $\theta$ is of course due to the fact that the model variance function used here involves by definition the single length $\theta$. This feature is realized, in some sense, by the roughness profiles. The argument is as follows.

Consider the $Z_{2}$ characteristic defined in Equation (3). If $y(x)$ is a random field, then the index $\left(Z_{2}\right)^{2}$ is a random variable. It can be easily shown that its expectation value is:

$$
\mathrm{E}\left[\left(\mathrm{Z}_{2}\right)^{2}\right]=-\sigma^{2} \rho^{\prime \prime}(0)=2 \pi \sigma^{2} / \theta^{2},
$$

The index $\left(Z_{2}\right)^{2}$ can be regarded as a statistic that estimates the value of the (a priori fixed) quantity $2 \pi \sigma^{2} / \theta^{2}$. In all, in regard of estimates:

$$
\mathrm{Z}_{2} \propto \sigma / \theta,
$$

It is also reminded that $Z_{2}$ correlates very well with the roughness coefficient JRC, which must be related to both the macro- and micro-scale of the profile. This recalls the almost perfect correlations found by Tse and Cruden [3], Yang et al. [12] and Yu and Vayssade [7]. However, $Z_{2}$ is rather related more to the micro-scales of the profile than to the macro-scales, due to the derivative involved in its definition. Therefore, it is concluded that, first, a single length may be adequate when modelling 
a roughness profile with a random field and second, that the quantity $\sigma / \theta$ is a good candidate for quantifying the roughness coefficient JRC in the framework of the random fields.

\section{Results}

\subsection{The $\sigma / \theta$ Ratio as an Estimate for JRC Coefficient}

Clearly, a direct way to determine $\sigma$ and $\theta$ for a given profile is needed. In this respect, the function $A C F(\Delta x)$ defined above must first be calculated. This is a priori even function that needs to be determined for $\Delta x$ in the interval $(0, L / 2)$. The point-variance $\sigma^{2}$ is estimated by $A C F(0)$, whilst $\theta$ is extracted from the normalized autocorrelation function $A C F(\triangle x) / A C F(0)$.

Explicitly, these quantities are constructed from the profile as follows. As mentioned above the sampled profile is a sequence of $N=1024$ numbers: $y_{n}$ for $n=0,1, \ldots, 1023$. The Fourier transform $Y_{k}$ of the profile $\mathrm{y}_{n}$ is defined by:

$$
Y_{k}=\sum_{n=0}^{N-1} y_{n} e^{-i 2 \pi \frac{n k}{N}},
$$

where, $k=0,1, \ldots, N-1$ are the wave-numbers. Then, $\operatorname{ACF}(\Delta x)$ is the Fourier transform of the magnitude-square of $Y_{k}$ :

$$
A C F(\Delta x)=\sum_{k=0}^{N-1} \frac{\left|Y_{k}\right|^{2}}{N^{2}} e^{-i 2 \pi \frac{m k}{N}},
$$

The correlation distance $\Delta x$ is of course discrete and is evaluated at the points $m \cdot 10 / \mathrm{N} \mathrm{cm}$, where $m$ is an integer, having divided the $10 \mathrm{~cm}$ length of the profile into $N=1023$ equally spaced intervals. The $A C F(\Delta x)$ so defined is a periodic function of period $10 \mathrm{~cm}$. As mentioned above, $A C F(\Delta x)$ is an even function thus essentially exists in the interval $(0,5 \mathrm{~cm})$ of correlation distances.

The point-variance $\sigma^{2}$ of the ten standard profiles along with their actual JRC coefficients is given in Table 1.

Table 1. The point-variance of the ten standard profiles along with their actual joint roughness coefficient (JRC) coefficients.

\begin{tabular}{ccc}
\hline Profile No. & Real JRC & $\sigma^{2}\left(\mathbf{c m}^{2}\right)$ \\
\hline 1 & 0.4 & 0.0002092 \\
2 & 2.8 & 0.000372183 \\
3 & 5.8 & 0.000969378 \\
4 & 6.7 & 0.001708827 \\
5 & 9.5 & 0.006983672 \\
6 & 10.8 & 0.037012614 \\
7 & 12.8 & 0.016019078 \\
8 & 14.5 & 0.032283009 \\
9 & 16.7 & 0.013746304 \\
10 & 18.7 & 0.008309631 \\
\hline
\end{tabular}

The normalized autocorrelation functions $A C F(\triangle \mathrm{x}) / A C F(0)$ as function of the distance $\Delta \mathrm{x}$ from $0 \mathrm{~cm}$ to $5 \mathrm{~cm}$ are shown in Figure 2 (labelled by the number of the profile).

The very form of these functions is quite telling. It is observed, for example, that the profiles No. 5 and 6 i.e., corresponding to JRC in the range 8-10 and 10-12 respectively, hardly differ in terms of spatial variability, which is also a large scale one. Their essential difference was the magnitude of waviness, measured by the point-variance $\sigma^{2}$. This is essentially visible in the profiles. Less obvious is the fact that the JRC ranges 2-4 and 18-20 (profile No. 2 and 10 respectively) appear to correspond to similar spatial variability, the difference again lying in the point-variance. The value of the length $\theta$ for each profile was extracted by fitting the model (Gaussian) normalized variance function $\rho(\Delta x)$ given above to the normalized autocorrelation function $A C F(\triangle x) / A C F(0)$. 


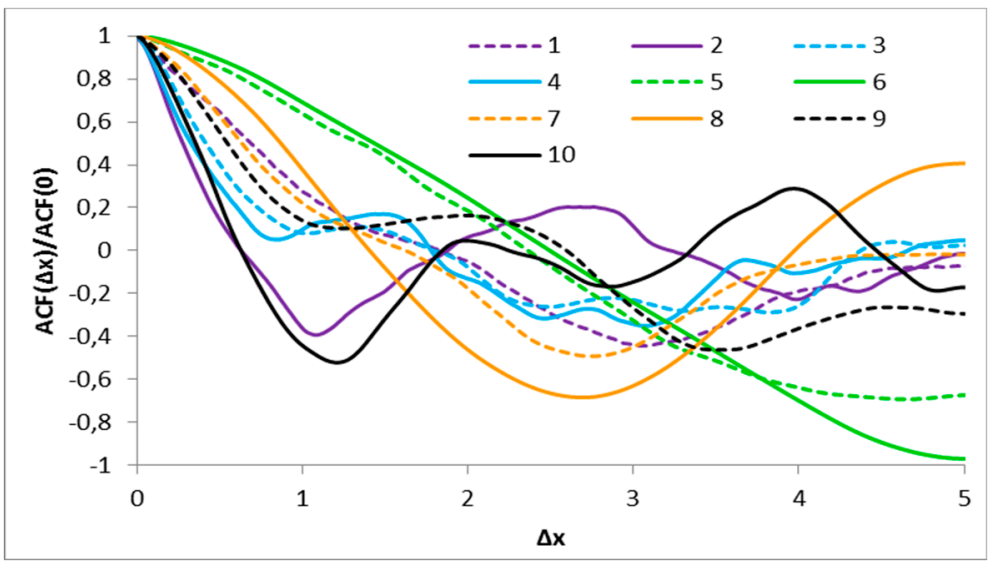

Figure 2. The normalized autocorrelation functions $A C F(\triangle x) / A C F(0)$ as function of the distance $\Delta x$ (labelled by the No. of Barton and Choubey's [4] profile; see also Appendix A).

The values of the spatial correlation length $\theta$ associated with each model function $\rho(\Delta x)$ are given in Table 2. These values result from the best fit of each $A C F(\Delta x) / A C F(0)$ by the respective model function $\rho(\Delta x)$. Along with them is given the ratio $\sigma / \theta$, which is our proposal for the JRC coefficient in the random field theory.

Table 2. The values of the spatial correlation length $\theta$ associated with each model function $\rho(\Delta x)$.

\begin{tabular}{ccc}
\hline Real JRC & $\boldsymbol{\theta}(\mathbf{c m})$ & $\boldsymbol{\sigma} / \boldsymbol{\theta}$ \\
\hline 0.4 & 1.497 & 0.0097 \\
2.8 & 0.599 & 0.0322 \\
5.8 & 0.952 & 0.0327 \\
6.7 & 0.763 & 0.0542 \\
9.5 & 2.540 & 0.0329 \\
10.8 & 2.731 & 0.0704 \\
12.8 & 1.383 & 0.0915 \\
14.5 & 1.596 & 0.1126 \\
16.7 & 1.216 & 0.0964 \\
18.7 & 0.684 & 0.1332 \\
\hline
\end{tabular}

Characteristic of the autocorrelation functions of the profiles is the negative part. Negative parts always occur in the autocorrelation function of a single realization, even if the random field belongs by construction to a specific type, e.g., Gaussian with Gaussian correlations. Therefore, if one is given a single realization of the field, the best way to proceed is by best fitting the obtained correlation/variance function to suitable model function. The Gaussian model correlation used here was chosen among various simple models (e.g., Markovian, white noise, triangular and cubic correlations; see [23]) as the one which performed the best. The quantity $\sigma / \theta$ is given in Table 2 for each roughness coefficient JRC. Moreover, the JRC- $\sigma / \theta$ relationship has been drawn in Figure 3. The R-squared of this relationship was only $3 \%$ smaller than the respective one given in Figure 1, also by the authors, for the respective JRC- $\log Z_{2}$ relationship. Both relationships have been extracted from the same figure (Barton and Choubey's [4]) without involving fine tuning of the digitization.

It should be noted that, not all of the waviness of the correlation functions, and their negative parts shown in Figure 2, need to be an artifact of the single realization. The cases of JRC 8-10, 10-12 and 14-16 exhibited a more deterministic tendency for negative values and a different pattern than most of the other cases. Clearly, the single-parameter model variance function in Equation (14) is too simple to cover all forms of behavior exhibited by the profiles. A two- or three-parameter model function is required. This shall be the subject of future work. 


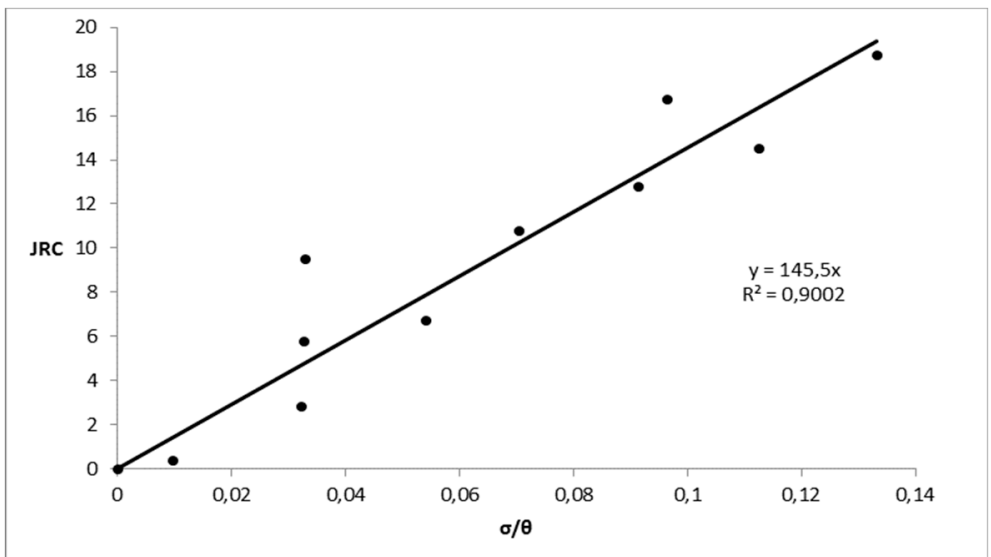

Figure 3. The JRC- $\sigma / \theta$ relationship.

\subsection{Stability of $\sigma / \theta$ as a Roughness Measure}

Estimating roughness from the image of a rock surface entails errors due to the quality of the image and its digitation. A simple test as to the behavior of the roughness measures is to calculate the index $Z_{2}$ and our index $\sigma / \theta$ and compare the results for the two cases of images. In this respect, the original JRC profiles given in Barton and Choubey [4] and the respective ones reproduced by Tse and Cruden [3] have been used. It is noted that, when the pages containing the standard JRC profiles are printed in A4 paper using the "actual size" option of printer, the linear scale (accompanying the profiles) of $10 \mathrm{~cm}$ in Barton and Choubey [4] measured (approximately) $6 \mathrm{~cm}$, whilst the same scale of $10 \mathrm{~cm}$ in Tse and Cruden [3] measured just $3 \mathrm{~cm}$. It is also reminded that, none of these raster images of the late 1970s meet the quality standards of today (e.g., the quality of vector images). The profiles of both images were analyzed in the exact same way. The results have been presented below.

The JRC- $\log Z_{2}$ relationships extracted by Tse and Cruden's [3] and Barton and Choubey's [4] image are shown together in Figure 4, the latter has also be drawn in Figure 1. From Figure 4, it is clear that, the different images have given incomparable estimates for the roughness. This is of particular importance because the same $Z_{2}$ value led to complete JRC values.

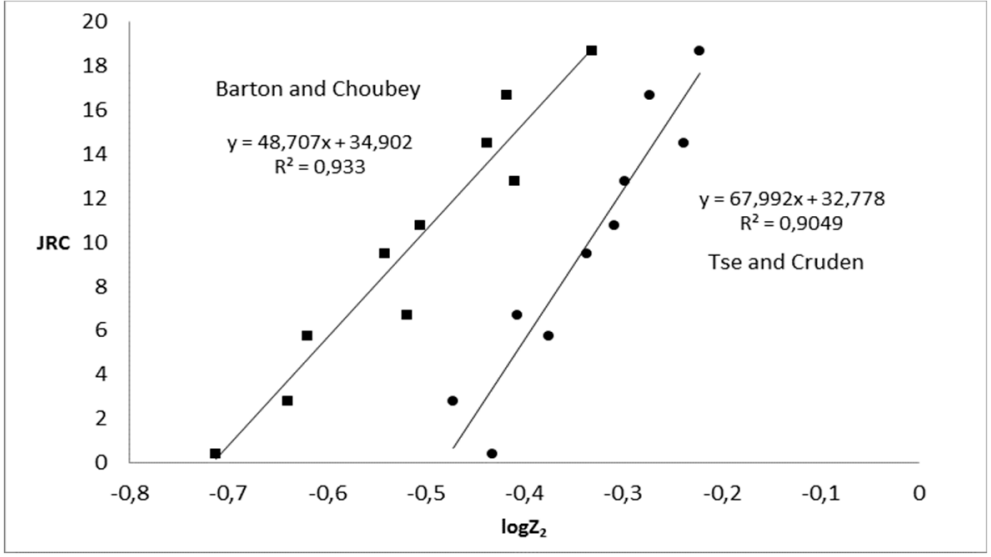

Figure 4. Graph comparing the JRC- $\log Z_{2}$ relationship extracted from Tse and Cruden's [3] and Barton and Choubey's [4] image.

The same has been done but for the JRC- $\sigma / \theta$ relationship. As shown in Figure 5, there is a much greater consistency between the estimates. The level of consistency is additionally shown by plotting the two sets of results against each other. Figure 6 shows the results for $\log Z_{2}$, whilst Figure 7 shows the results for the $\sigma / \theta$ index introduced in present work. For the case of $\sigma / \theta$, the higher $R^{2}$ value has been noticed, and also the proximity of the slope of the best fit to the value of 1 . The reason why the 
$\sigma / \theta$ index appears to be more stable than the index $Z_{2}$ might be described as follows. $Z_{2}$ contains the information contained in $A C F(\Delta x)$ for equal $\Delta x$ to the length of the interval between sampling points, i.e., the infinitesimal interval of the given digitation. On the other hand, $\theta$ is estimated employing the information contained in $A C F(\Delta x)$ over all meaningful correlation distances. Apparently, the latter feature operates as a regularizing factor smoothing those errors present in the $Z_{2}$ estimate.

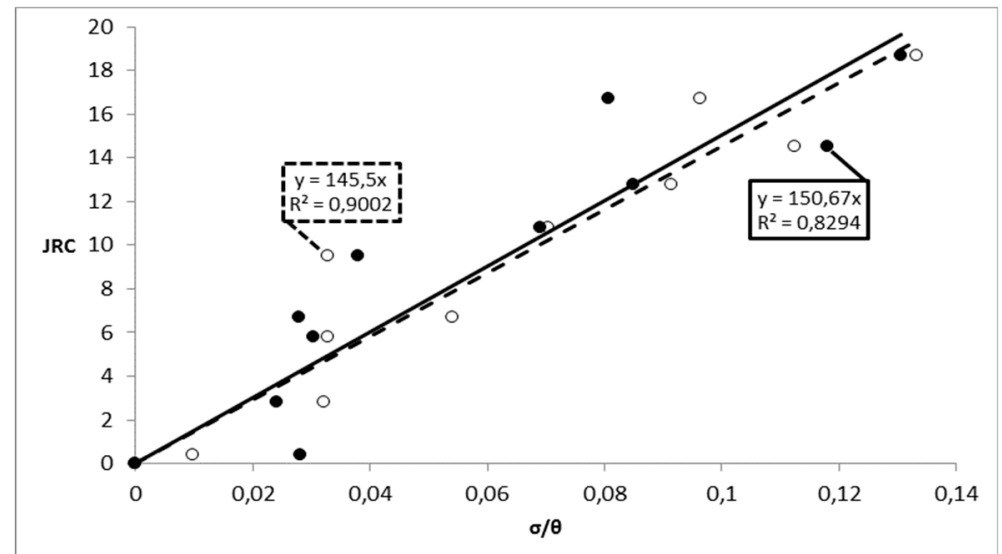

Figure 5. Graph comparing the JRC- $\sigma / \theta$ relationship extracted from Barton and Choubey's [4] and Tse and Cruden's [3] image (white and black dots respecrively).

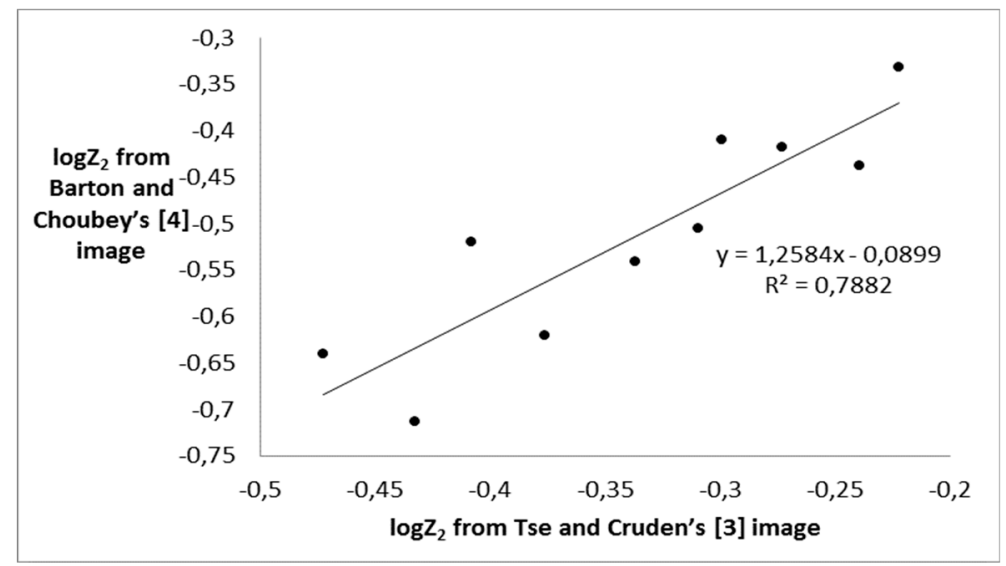

Figure 6. Graph comparing the $\log Z_{2}$ values obtained from Tse and Cruden's [3] and Barton and Choubey's [4] image.

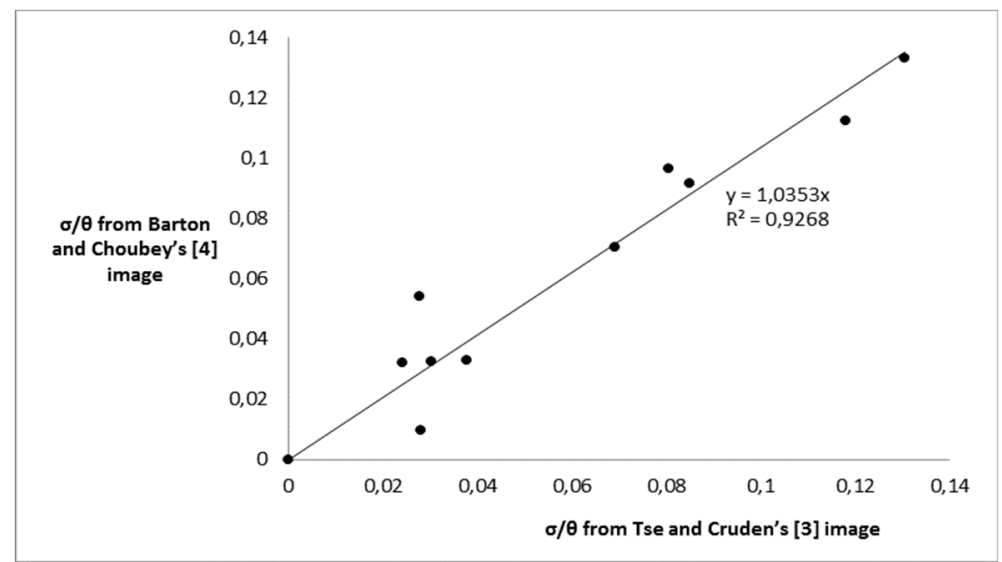

Figure 7. Graph comparing the $\sigma / \theta$ values obtained from Tse and Cruden's [3] and Barton and Choubey's [4] image. 


\subsection{Obtaining $\theta$ through JRC}

As mentioned earlier, this work intends to embed the estimation of the joint roughness coefficient in the framework of random fields. In this respect, JRC is estimated through $\sigma$ and $\theta$, or better through the $\sigma / \theta$ ratio. However, the present work could also be useful in an advanced probabilistic rock slope stability analysis [26] based on random fields, considering that the Barton-Bandis criterion [27] has been adopted. In order for a random field analysis to be performed, the spatial correlation length $\theta$ of joint roughness must be known. If now the JRC coefficient and the variance $\sigma$ of the profile are known, the spatial correlation length $\theta$ can be obtained from the best fit relation of Figure 3, as follows:

$$
\Theta=145.5 \sigma / \mathrm{JRC}
$$

The JRC coefficient can be obtained directly through tilt tests, push or pull tests, or matching roughness profiles [4], whilst the variance $\sigma$ can be obtained from inspection of the digitized profile.

\section{Discussion}

The authors revisited the problem of determining the joint roughness coefficient (JRC) included in the Barton-Bandis failure criterion from the point of view of digitalized profiles of discontinuities. However, numerous indices have been correlated with JRC, with the geometric characteristic $Z_{2}$ being, probably, the most popular one. Based on the international literature, the statistical measure $S F$ (Structure Function), has been a good alternative giving also very strong correlations with JRC.

As the random fluctuation of discontinuities can be considered as being random fields, the present paper targeted on embedding the estimation of the joint roughness coefficient (JRC) in the framework of the theory of random fields. The analysis showed that the fluctuation of discontinuities in both micro- and macro scale was related to a single length and more specifically to the spatial correlation length, $\theta$ (essential parameter in the theory of random fields). Indeed, it was found that the quantity $\sigma / \theta$ was a good candidate for quantifying the roughness coefficient JRC. Usually, the determination of $\theta$ relies on the best fit of a suitably chosen model variance function to the normalized autocorrelation function. The stability of the proposed $\sigma / \theta$ index as a roughness measure was compared against the characteristic $Z_{2}$. In this respect, the two indices were applied to images of the same discontinuity profiles and more specifically, the ten standard JRC profiles given by Barton and Choubey [4], but of different quality. From the results, it is clear that the estimates for JRC by the proposed method appear to be much more stable, in contrast to $Z_{2}$ or, as explained earlier, $S F$ (structure function).

The fact that the $R$-squared value for the JRC- $\sigma / \theta$ relationship of Figure $3\left(R^{2}=0.9002\right)$, although very strong, was not perfectly strong can be attributed to three main reasons. First, no fine tuning of the digitization of Barton and Choubey's [4] figure was involved. Second, the ten JRC profiles were representative of the two-dimensional cross-sections, whilst their JRC values were average values referring to the respective three-dimensional fluctuating surface of the rock blocks. Third, despite the versatility of the random field method, the derived $\theta$ values depended on the extend of the sampling domain $[28,29]$. An extensive parallel research conducted by the authors (paper under preparation) indicated that, for eliminating this kind of error in the proposed JRC- $\sigma / \theta$ relationship, a domain at least $35 \theta$ in length was required to be considered. Indeed, as the domain length increases (in this respect, the length of discontinuity) the $\theta$ value reaches asymptotically its maximum value for the specific random field. This is in agreement with the empirical scale correction relationship suggested by Barton and Bandis [30]:

$$
\mathrm{JRC}_{\mathrm{n}} \approx \mathrm{JRC}_{\mathrm{o}}\left(\frac{\mathrm{L}_{\mathrm{n}}}{\mathrm{L}_{\mathrm{o}}}\right)^{-0.02 \cdot \mathrm{JC}_{\mathrm{o}}}
$$

where, $J R C_{n}$ and $J R C_{o}$ are the joint roughness coefficients corresponding to samples of length $L_{n}$ and $\mathrm{L}_{\mathrm{o}}=10 \mathrm{~cm}$ respectively. More specifically, Equation (22) shows that, the $\mathrm{JRC}_{\mathrm{n}}$ value decreases as the $L_{n} / L_{o}$ ratio increases towards a minimum asymptotic value. On the other hand, as $L_{n} / L_{o}$ increases, the $\sigma / \theta$ ratio and thus, the JRC value decreases (recall the JRC- $\sigma / \theta$ relationship in Figure 3) 
towards a minimum asymptotic value. Based on the $\theta$ values given in Table 2, the rock joint profiles should be at least 2 to 9 times longer than the $10 \mathrm{~cm}$ of the original Barton and Choubey's [4] profiles (value depending on the fluctuation pattern of each profile) for effectively determining the JRC.

The above has been supported by the experimental findings of Barton and Choubey [4]. In this respect, Barton and Choubey performed three tilt tests on a $40 \times 45 \mathrm{~cm}$ joint area (sliding along the long dimension) and they found an (average) joint roughness coefficient value equal to 5.5. The same sample was sawn by Barton and Choubey into 18 samples $4.9 \times 9.8 \mathrm{~cm}$ in size (samples by $45 / 9.8=4.6$ times shorter in the shear direction than the original joint area). The shear direction was marked so that each of the small specimens could be tilt-tested, push-tested and shear box tested in the same direction as the original tilt test of the $45 \mathrm{~cm}$ long joint. The results of this investigation are summarized in Table 3. From the table in question it is inferred that: (i) The (mean) JRC value depends greatly on the type of test; (ii) the standard deviation alone is not a good index for indicating the proper type of test; and (iii) the best estimate was obtained from the tilt test (please compare the $\mathrm{JRC}_{\mathrm{n}}=5.12$ value with the $J R C_{n}=5.5$ for the $40 \times 45 \mathrm{~cm}$ joint area). The latter could be attributed to test compatibility, as the $40 \times 45 \mathrm{~cm}$ joint area was also tested on a tilt test configuration.

Table 3. JRC values obtained by different test methods (see Barton and Choubey [4]) and correction for scale.

\begin{tabular}{ccccc}
\hline Statistical Description of Test Data and JRCn Values & Shear Box $^{\mathbf{1}}$ & Tilt $^{\mathbf{1}}$ & Push $^{\mathbf{1}}$ & Overall $^{\text {Des }}$ \\
\hline Number of samples tested & 18 & 6 & 12 & 36 \\
Mean JRC value, $\mu$ & 8.69 & 6.18 & 10.17 & 8.76 \\
Standard deviation, $\sigma$ & 1.18 & 0.66 & 0.55 & 1.63 \\
$\mathrm{JRC}_{\mathrm{n}}{ }^{2}$ (Equation (22)) & 6.67 & 5.12 & 7.46 & 6.71 \\
\hline
\end{tabular}

${ }^{1}$ Not the same samples used in the tilt test were used in the push test, but all samples were tested in the shear box.
2 Reduction of the above JRC values to samples $45 \mathrm{~cm}$ in length based on Barton and Bandis's [30] scale correction.

For considering the extent of the pattern of fluctuations on the JRC value, the authors suggest that the JRC be determined based on joint profiles of adequate length. This suggestion is independent of the method adopted (i.e., determination of JRC in the field, in the laboratory or based on correlations e.g., with $\mathrm{Z}_{2}$ or $\mathrm{SF}$ ). For discontinuities with large scale waviness, apparently, this length should be even greater. A test joint surface length is adequate when considering greater length, as the JRC value remains practically the same.

It is noted that, if the present method is adopted, no correction for the influence of scale on JRC (such as the one proposed by Barton and Bandis [30]; see Equation (22)), is required. However, because of the greater possibility of weaknesses in a large surface, it is likely that the average joint wall compressive strength (JCS) decreases with increasing scale. In this respect, Barton and Bandis' [30] scale correction for JCS (recall Equation (6)) is suggested. It is additionally mentioned that, Barton and Bandis's [30] scale correction was derived from shear tests over a ten-fold range of block sizes, involving linear extrapolation outside the block size range $0.1 \mathrm{~m}$ to $1 \mathrm{~m}$. On the contrary, the proposed method is straightforward, not involving such procedures.

Finally, as the problem is clearly one dimensional, and since sliding will take place along a specific direction, the final JRC value should be an average value derived from a number of profiles parallel to the direction of sliding. In this respect, the JRC $=145.5 \sigma / \theta$ relationship is a convenient and reliable tool for such an analysis. Refinement of this relationship based on longer sampling domain lengths is subject matter of future research by the authors, although it is believed that this best fit equation will not dramatically change.

Author Contributions: Conceptualization, E.G. and L.P.; methodology, E.G.; software, E.G.; validation, E.G. and L.P.; formal analysis, E.G.; writing—original draft preparation, E.G. and L.P.; writing—review and editing, E.G. and L.P.; visualization, E.G. and L.P.; supervision, L.P.; funding acquisition, L.P.

Funding: This research was funded by the Cyprus University of Technology, grant number EX-20081. 
Conflicts of Interest: The authors declare no conflicts of interest. The funders had no role in the design of the study; in the collection, analyses, or interpretation of data; in the writing of the manuscript, or in the decision to publish the results.

\section{Appendix A}

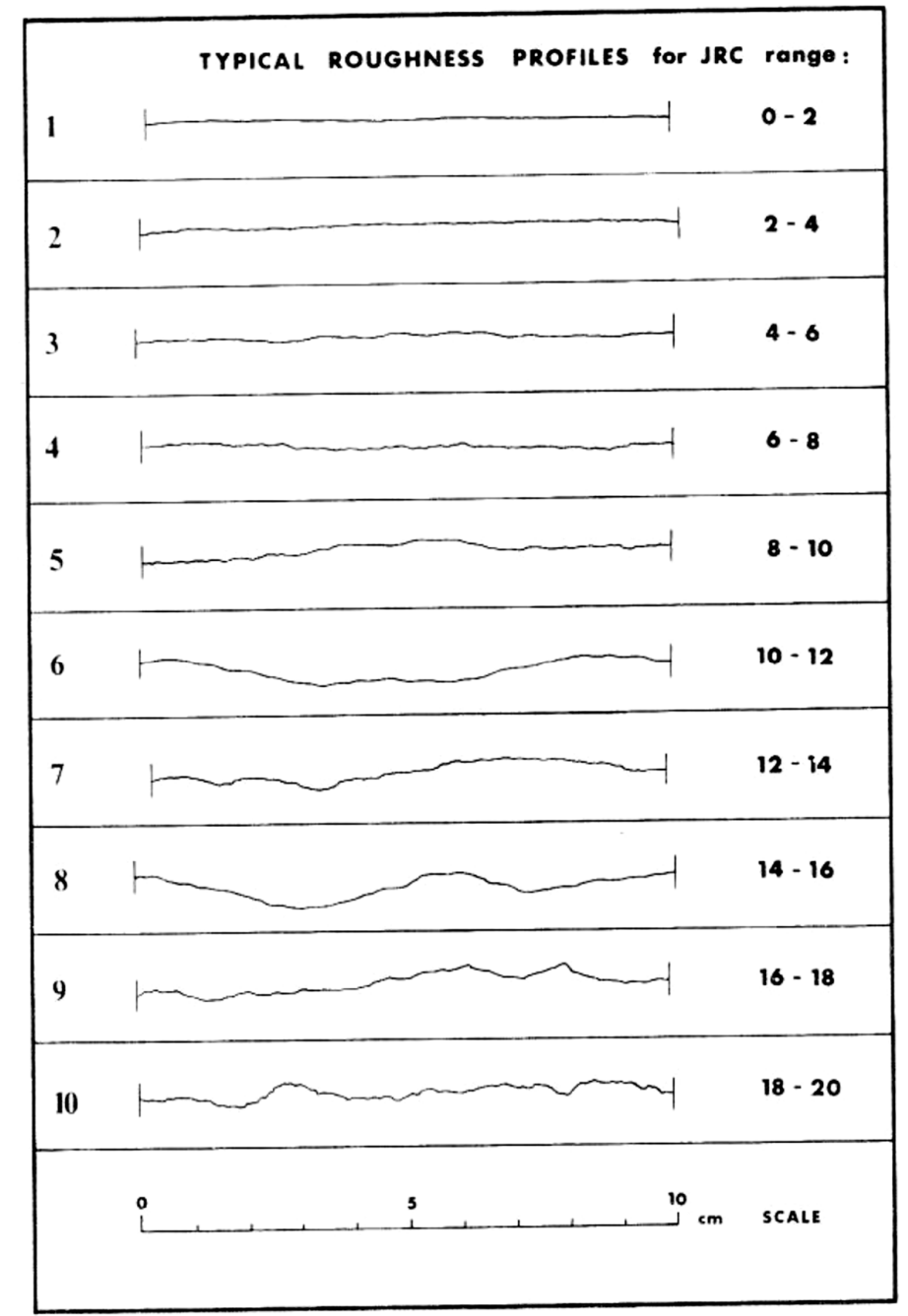

Figure A1. The ten standards profiles given by Barton and Choubey [4].

\section{References}

1. American Society of Mechanical Engineers. Surface Roughness, Waviness and Lay; ASA B46.1; American Society of Mechanical Engineers: New York, NY, USA, 1955.

2. Myers, N.O. Characterization of surface roughness. Wear 1962, 5, 182-189. [CrossRef]

3. Tse, R.; Cruden, D.M. Estimating Joint Roughness Coefficients. In Proceedings of the International Journal of Rock Mechanics and Mining Sciences Geomechanics Abstracts; Elsevier: Amsterdam, The Nederland, 1979; Volume 16, pp. 303-307.

4. Barton, N.; Choubey, V. The shear strength of rock joints in theory and practice. Rock Mech. 1977, 10, 1-54. [CrossRef]

5. Ulusay, R. (Ed.) The ISRM Suggested Methods for Rock Characterization, Testing and Monitoring: 2007-2014; Springer International Publishing: Cham, Switzerland, 2015; ISBN 978-3-319-07712-3.

6. Sayles, R.S.; Thomas, T.R. The spatial representation of surface roughness by means of the structure function: A practical alternative to correlation. Wear 1977, 42, 263-276. [CrossRef]

7. Yu, X.; Vayssade, B. Joint profiles and their roughness parameters. Int. J. Rock Mech. Min. Sci. Geomech. Abstr. 1991, 28, 333-336. [CrossRef] 
8. Gao, Y.; Wong, L.N.Y. A Modified Correlation Between Roughness Parameter Z 2 and the JRC. Rock Mech. Rock Eng. 2015, 48, 387-396. [CrossRef]

9. Turk, N.; Greig, M.; Dearman, W.; Amin, F.F. Characterization of Rock Joint Surfaces by Fractal Dimension. In Proceedings of the 28th U.S. Symposium on Rock Mechanics (USRMS), Tucson, AZ, USA, 29 June-1 July 1987; Association, A.R.M., Ed.; A. A. Balkema: Rotterdam, The Netherlands, 1987; p. ARMA-87-1223.

10. Lamas, L.N. An experimental and analytical study of the roughness of granite joints. In Proceedings of the ISRM International Symposium-EUROCK 96, Turin, Italy, 2-5 September 1996; International Society for Rock Mechanics and Rock Engineering; A. A. Balkema: Rotterdam, The Netherlands, 1996.

11. Chen, S.J.; Zhu, W.C.; Zhang, M.S.; Yu, Q.L. Fractal description of rock joints based on digital image processing technique. Chin. J. Geotech. Eng. 2012, 34, 2087-2092.

12. Yang, Z.Y.; Lo, S.C.; Di, C.C. Reassessing the Joint Roughness Coefficient (JRC) Estimation Using Z2. Rock Mech. Rock Eng. 2001, 34, 243-251. [CrossRef]

13. Li, Y.; Zhang, Y. Quantitative estimation of joint roughness coefficient using statistical parameters. Int. J. Rock Mech. Min. Sci. 2015, 77, 27-35. [CrossRef]

14. Li, Y.; Huang, R. Relationship between joint roughness coefficient and fractal dimension of rock fracture surfaces. Int. J. Rock Mech. Min. Sci. 2015, 75, 15-22. [CrossRef]

15. Carr, J.R.; Warriner, J.B. Rock mass classification of joint roughness coefficient. In Proceedings of the 28th US Rock Mechanics Symposium, Tucson, AZ, USA, 29 June-1 July 1987; pp. 72-86.

16. Bae, D.; Kim, K.; Koh, Y.; Kim, J. Characterization of Joint Roughness in Granite by Applying the Scan Circle Technique to Images from a Borehole Televiewer. Rock Mech. Rock Eng. 2011, 44, 497-504. [CrossRef]

17. Askari, M.; Ahmadi, M. Failure Process after Peak Strength of Artificial Joints by Fractal Dimension. Geotech. Geol. Eng. 2007, 25, 631-637. [CrossRef]

18. Shitian, Q.S.Z.Z.W.; Runqiu, H. Fractal characteristics of jointed rock mass and its engineering geology significance. J. Eng. Geol. 1993, 2, 14-23.

19. Jia, H.Q. Experimental Research on Joint Surface State and the Characteristics of Shear Failure. Ph.D. Thesis, Central South University, Changsha, China, 2011.

20. Zhou, C.B.; Xiong, W.L. Relation between joint roughness coefficient and fractal dimension. J. Wuhan Univ. Hydraul. Electr. Eng. 1996, 29, 1195-1197.

21. Maerz, N.H.; Franklin, J.A. Roughness scale effect and fractal dimension. In Proceedings of the 1st International Workshop on Scale Effects in Rock Masses, Loen, Norway, 7-8 June 1990; A. A. Balkema: Rotterdam, The Netherlands, 1990; pp. 121-126.

22. Liu, S.Y. Fractional dimension method of JRC measurement. Investig. Sci. Technol. 1993, 6, $26-29$.

23. Fenton, G.; Griffiths, D.V. Risk Assessment in Geotechnical Engineering; Wiley: New York, NY, USA, 2008; ISBN 9780470178201.

24. Vanmarcke, E.H. Random Fields: Analysis and Synthesis; MIT Press: Cambridge, MA, USA, 1983.

25. Batchelor, G.K. (Ed.) The Theory of Homogeneous Turbulence; Cambridge Monographs on Mechanics and Applied Mathematics; Cambridge University Press: Cambridge, UK, 1953.

26. Gravanis, E.; Pantelidis, L.; Griffiths, D.V. An analytical solution in probabilistic rock slope stability assessment based on random fields. Int. J. Rock Mech. Min. Sci. 2014, 71, 19-24. [CrossRef]

27. Barton, N.; Bandis, S. Review of predictive capabilities of JRC-JCS model in engineering practice. In Proceedings of the International Symposium on Rock Joints, Loen, Norway, 4-6 June 1990; Barton, N., Stephenson, O., Eds.; CRC Press Book; A. A. Balkema: Rotterdam, The Netherlands, 1990; pp. 603-610.

28. Nie, X.; Zhang, T.; Huang, H.; Liu, Z.; Lacasse, S. Scale of Fluctuation for Geotechnical Probabilistic Analysis; IOS Press: Rotterdam, The Netherlands, 2015; pp. 816-821.

29. Fenton, G.A. Random field modeling of CPT data. J. Geotech. Geoenviron. Eng. 1999, 125, 486-498. [CrossRef]

30. Barton, N.; Bandis, S. Effects of block size on the shear behavior of jointed rock. In Proceedings of the 23rd US Symposium on Rock Mechanics (USRMS), Berkeley, CA, USA, 25-27 August 1982; American Rock Mechanics Association: Richardson, TX, USA, 1982; pp. 739-760.

(C) 2019 by the authors. Licensee MDPI, Basel, Switzerland. This article is an open access article distributed under the terms and conditions of the Creative Commons Attribution (CC BY) license (http://creativecommons.org/licenses/by/4.0/). 